\title{
Human Retrosplenial Cortex Displays Transient Theta Phase Locking with Medial Temporal Cortex Prior to Activation during Autobiographical Memory Retrieval
}

\author{
Brett L. Foster, ${ }^{\star}$ Anthony Kaveh, ${ }^{\star}$ Mohammad Dastjerdi, Kai J. Miller, and Josef Parvizi \\ Laboratory of Behavioral \& Cognitive Neurology, Stanford Human Intracranial Cognitive Electrophysiology Program, Department of Neurology \& \\ Neurological Sciences, Stanford University, Stanford, California 94305
}

\begin{abstract}
The involvement of retrosplenial cortex (RSC) in human autobiographical memory retrieval has been confirmed by functional brain imaging studies, and is supported by anatomical evidence of strong connectivity between the RSC and memory structures within the medial temporal lobe (MTL). However, electrophysiological investigations of the RSC and its interaction with the MTL have mostly remained limited to the rodent brain. Recently, we reported a selective increase of high-frequency broadband $(\mathrm{HFB} ; 70-180 \mathrm{~Hz})$ power within the human RSC during autobiographical retrieval, and a predominance of $3-5 \mathrm{~Hz}$ theta band oscillations within the RSC during the resting state. In the current study, we aimed to explore the temporal dynamics of theta band interaction between human RSC and MTL during autobiographical retrieval. Toward this aim, we obtained simultaneous recordings from the RSC and MTL in human subjects undergoing invasive electrophysiological monitoring, and quantified the strength of RSC-MTL theta band phase locking. We observed significant phase locking in the $3-4 \mathrm{~Hz}$ theta range between the RSC and the MTL during autobiographical retrieval. This theta band phase coupling was transient and peaked at a consistent latency before the peak of RSC HFB power across subjects. Control analyses confirmed that theta phase coupling between the RSC and MTL was not seen for other conditions studied, other sites of recording, or other frequency ranges of interest $(1-20 \mathrm{~Hz})$. Our findings provide the first evidence of theta band interaction between the human RSC and MTL during conditions of autobiographical retrieval.
\end{abstract}

\section{Introduction}

The ability to memorize new environments, and the events associated with them, provides an important survival function. The neural systems underlying this adaptive behavior have been identified in growing detail in the rodent brain (Buzsáki, 2005). While the hippocampus and medial temporal lobe (MTL) cortical structures show direct coding for spatial and episodic information (Bird and Burgess, 2008), it is the retrosplenial cortex (RSC) that has been implicated as a putative convergent region (Damasio, 1989), essential to constructing coherent representations for retrieving and navigating previously experienced events and environments (Epstein, 2008; Vann et al., 2009; Ranganath and Ritchey, 2012). In line with these findings, anatomical tracing studies in non-human primates (Kobayashi and Amaral, 2003; Parvizi et al., 2006; Aggleton et al., 2012), as well as human brain

Received Feb. 1, 2013; revised May 11, 2013; accepted May 16, 2013.

Author contributions: B.L.F., M.D., and J.P. designed research; B.L.F., A.K., M.D., K.J.M., and J.P. performed research; B.L.F., A.K., M.D., and K.J.M. contributed unpublished reagents/analytic tools; B.L.F., A.K., and K.J.M. analyzed data; B.L.F., A.K., and J.P. wrote the paper.

This research was funded by National Institutes of Health R01 NS078396-01 and the Stanford NeuroVentures Program to J.P. We thank the patients for their involvement in this study, Stanford Epilepsy Monitoring Unit staff for assisting with patient recordings, and Vinitha Rangarajan and Kevin Weiner for comments on this manuscript.

The authors declare no competing financial interests.

*B.L.F. and A.K. contributed equally to this work.

Correspondence should be addressed to Dr. Josef Parvizi, Department of Neurology and Neurological Sciences, 300 Pasteur Drive, Stanford, CA, 94305. E-mail: jparvizi@stanford.edu.

DOI:10.1523/JNEUROSCI.0513-13.2013

Copyright $\odot 2013$ the authors $\quad 0270-6474 / 13 / 3310439-08 \$ 15.00 / 0$ imaging studies (Greicius et al., 2009; Margulies et al., 2009), show a strong reciprocal relationship between the RSC and MTL.

To date, our understanding of RSC and MTL interactions in the human brain is predominantly derived from neuroimaging studies, which provide limited information about the temporal dynamics of interaction. While electrophysiological recordings can provide higher temporal resolution, such recordings from the human RSC have only been possible with an invasive intracranial approach. The anatomical location of the RSC-hidden within the medial cortical surface closely tucked under the splenium of the corpus callosum as its name infers-makes it inaccessible to noninvasive explorations with scalp electroencephalography.

Recently, we have begun to explore the electrophysiological dynamics of the human RSC (Dastjerdi et al., 2011; Foster et al., 2012), as part of a wider intracranial investigation into the human posteromedial cortex (PMC) (Parvizi et al., 2006). Extending previous electrophysiological evidence suggesting a suppression of neural activity in the human PMC during externally directed attentional effort (Miller et al., 2009; Jerbi et al., 2010; Dastjerdi et al., 2011; Ossandón et al., 2011), we recently reported that human RSC displays a clear suppression of activity during simple arithmetic calculations, but electrophysiological activation during conditions of autobiographical episodic memory retrieval (Foster et al., 2012). Consistent with previous literature (Lachaux et al., 2012), these cortical responses were best captured by a highfrequency broadband (HFB) power range $(\sim 70-180 \mathrm{~Hz})$. In ad- 

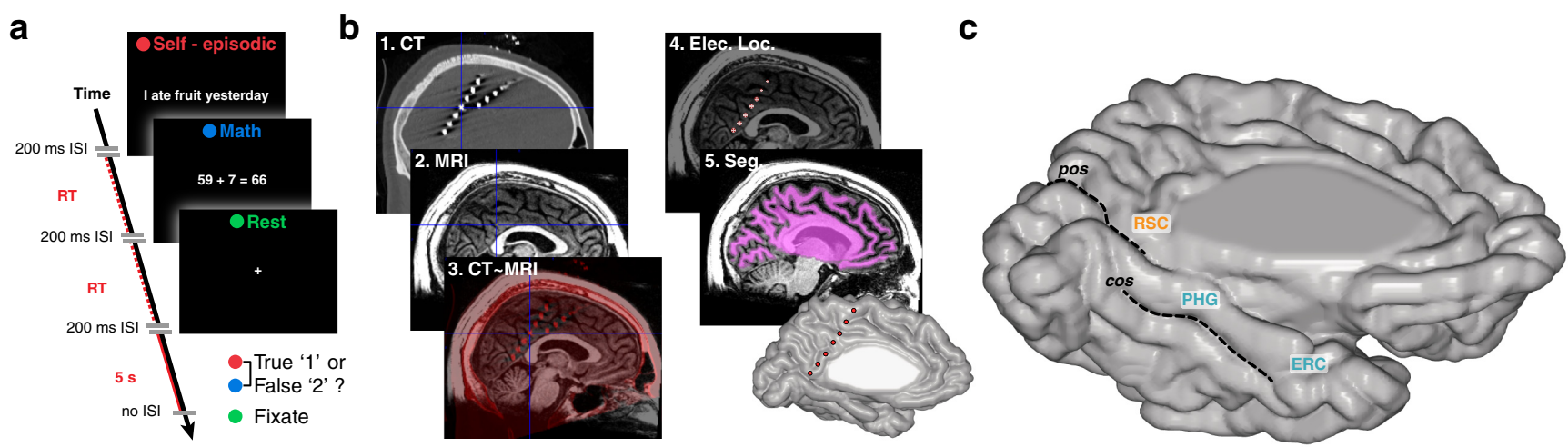

Figure 1. Experimental task and electrode localization. $\boldsymbol{a}$, All subjects performed a simple behavioral task requiring true/false (button press "1" $/$ " 2 ") judgments of either autobiographical statements (self-episodic) or arithmetic equations (math) as previously described (Foster et al., 2012). These conditions had a variable RT duration and were randomly interleaved by a fixed $5 \mathrm{~s}$ rest period (rest). The interstimulus interval (ISI) was $200 \mathrm{~ms}$; however, no ISI followed the occurrence of rest trials. $\boldsymbol{b}$, Intracranial electrodes were localized in each subject by aligning post implant CT images with preoperative higher resolution MRl images, and then projecting electrode coordinates to the segmented cortical surface (Hermes et al., 2010). Adapted from Foster et al., 2012 with permission. c, Electrodes were anatomically classified in each subject using common sulcal and gyral landmarks on the ventromedial surface. pos, parieto-occipital sulcus; cos, collateral sulcus; RSC, retrosplenial cortex; PHG, parahippocampal gyrus; ERC, entorhinal cortex (Parvizi et al., 2006; Weiner and Grill-Spector, 2013).

dition, we have also demonstrated that human RSC displays theta band oscillations $(3-5 \mathrm{~Hz})$ during rest, which in turn phase modulate local HFB amplitude (Foster and Parvizi, 2012). This theta phase modulation reflects an important oscillatory motif seen throughout the MTL of many species including humans, which may be important for organizing neural dynamics during conditions of learning and memory (Buzsáki, 2005; Fell and Axmacher, 2011).

In light of extant data, the present study aimed to explore task-related theta phase locking between RSC and MTL in human subjects. We hypothesized that this coupling would be specific to the theta band range during conditions of autobiographical memory retrieval, and therefore not be observed for other frequencies, conditions, or locations studied.

\section{Materials and Methods}

Subjects. Intracranial recordings from four subjects (mean age $\pm \mathrm{SD}=$ $32 \pm 12$ years) with refractory epilepsy are reported here. These subjects come from a larger cohort previously reported (Foster et al., 2012) and were selected because of their unique simultaneous electrode coverage over the RSC and MTL, as required for our specific aim. All subjects (S1-S4) were implanted with cortical surface electrodes. Depth recordings from the MTL (e.g., hippocampus) were not acquired. This unique sample is balanced for gender (female/male $=2 / 2$ ), hemisphere (right $(\mathrm{R}) /$ left $(\mathrm{L})=2 / 2)$, and cross-balanced for hemisphere within gender (female $=1 \mathrm{~L} / 1 \mathrm{R} ;$ male $=1 \mathrm{~L} / 1 \mathrm{R})$. Disease foci differed between subjects, with S2 and S3 having epileptic foci in the right and left MTL, whereas S1 and S4 had occipital and insular epileptic foci, respectively. All subjects were implanted as part of a clinical assessment for neurosurgical resection at Stanford Medical Center, and provided written informed consent to participate in the study described below, which was approved by the Stanford Institutional Review Board.

Data recording and experimental task. Electrocorticographic (ECoG) potentials were recorded subdurally from the cortical surface with strip and grid electrodes (Adtech Medical Instruments), using a multichannel research system (Tucker Davis Technologies). ECoG signals were acquired at a sampling rate of $3052 \mathrm{~Hz}$ and with a $0.5-300 \mathrm{~Hz}$ bandpass range. All recordings were referenced to a clinically selected ECoG electrode and were subsequently re-referenced for data analysis (described below). Stimulus events were simultaneously recorded by the same system using a TTL pulse input triggered by a photodiode sensor attached to the display of the presentation laptop. As previously described (Foster et al., 2012), ECoG signals were continuously recorded and time locked to the performance of a cognitive task that robustly modulates HFB activity in the RSC. In brief, this task requires true/false judgments (button press
"1" or "2") of episodic autobiographical statements (self-episodic condition) or arithmetic equations (math condition), which are randomly interleaved with periods of fixation (rest condition; Fig. 1a). For example, all subjects responded to the same set of self-episodic sentences such as "I ate fruit yesterday" or "I drank coffee this morning," and simple arithmetic equations such as " $59+7=66$ " or " $8+65=73$ " (for all stimuli see Foster et al., 2012). Although subjects provided true or false judgments for the autobiographical statements, we did not retrospectively investigate their veracity, which can be difficult to objectively ascertain for many of the stimuli.

Data analysis. In light of previous evidence for selective event-related electrophysiological changes in RSC during the task conditions described above (Foster et al., 2012), we sought to relate the dynamics of this response to the MTL. To do this, we studied subjects with simultaneous electrode coverage over both RSC and MTL. Electrode locations were confirmed and classified using both 2D CT-MRI fused images viewed orthogonally and by high-resolution 3D cortex-electrode reconstructions (Hermes et al., 2010; Foster et al., 2012) (Fig. 1b). In the current context, recording sites medial to the collateral sulcus constituted the "MTL" cortices whereas sites proximal to the splenium of the corpus callosum (BA 29/30) and dorsal to the parahippocampal gyrus (PHG) constituted the RSC, as described in our previous work (Parvizi et al., 2006; Foster et al., 2012) (Fig. 1c). We used bipolar referencing (see below) and labeled each pair of electrodes as being in the RSC or MTL if both electrodes or only one electrode of the pair was within these anatomical regions of interest.

We aimed to test the hypothesis that RSC would display selective theta band phase coupling with the MTL only during autobiographical memory retrieval (self-episodic condition). To support the validity of this prediction, we used surrogate data, and applied three domains of control to verify that our results were specific to the following: (1) the selfepisodic condition (task control), (2) the RSC-MTL (region control), and (3) the theta band range (frequency control).

Before time-frequency analysis, the data was re-referenced to sequential next-neighbor bipolar pairs (e.g., A-B, B-C, C-D...) from within electrode strips, and when appropriate, between electrode strips of approximately the same center-to-center interelectrode distance $(\sim 10 \mathrm{~mm}$; Fig. 2a). Although common average referencing is a standard practice for the analysis of ECoG data, bipolar referencing is commonly used to greatly reduce the spurious influence of volume conduction and shared reference upon estimates of temporal synchrony (Nunez et al., 1997; Lachaux et al., 2003; Niedermeyer and Lopes da Silva, 2005). As depicted in Figure 2, bipolar referencing is simply the pointwise subtraction of two recorded time series, producing a common mode rejection of shared temporal features (e.g., hardware drift, reference drift, line noise, etc.) that may exist across all recording sites and therefore produce spurious 

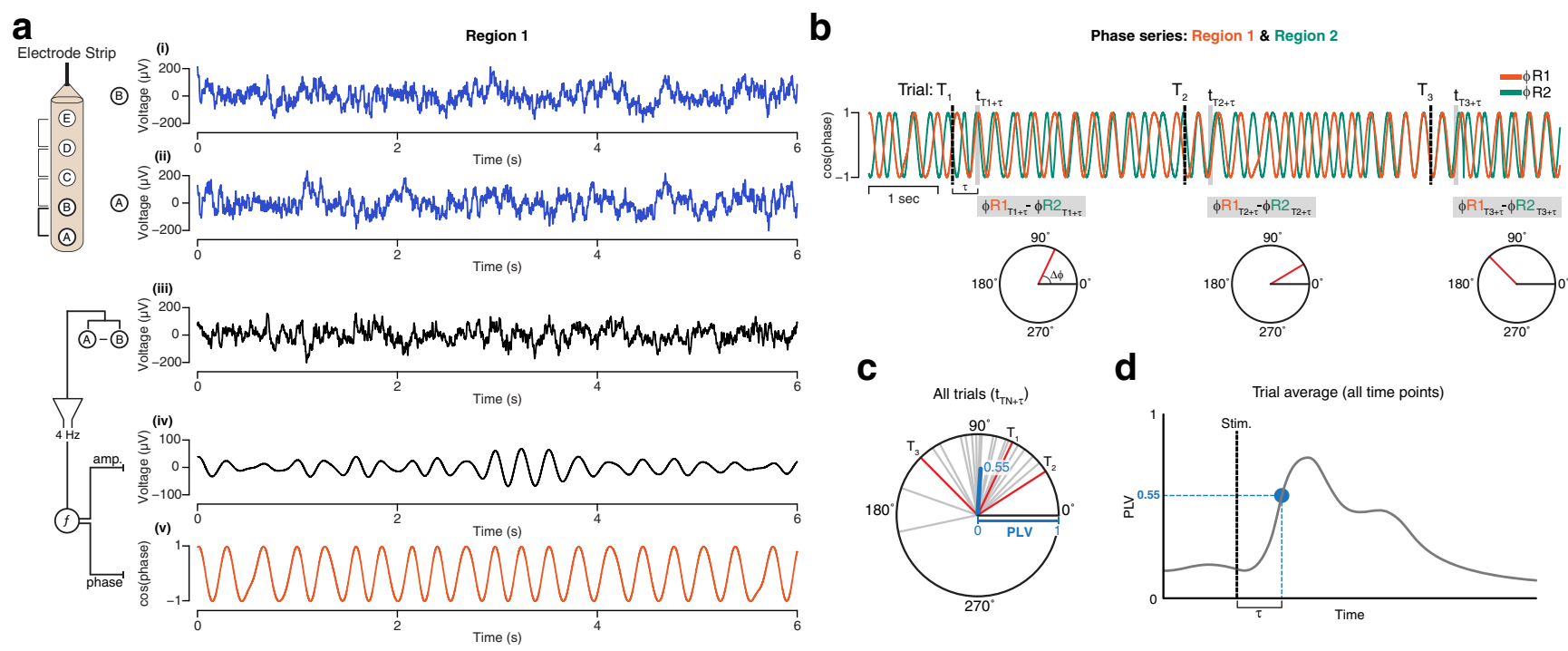

Figure 2. Data analysis. $\boldsymbol{a}$, Using subdural electrodes (e.g., linear strip arrays) raw ECoG signals (voltage) are recorded from each electrode (i-ii; electrodes A and B), initially referenced to a clinically chosen site elsewhere. These raw signals are then pairwise re-referenced using a sequential next-neighbor bipolar subtraction, which is simply the pointwise voltage difference between two adjacent electrodes (iii; e.g., electrode A- electrode B, B-C, C-D, D-E). Each differentiated channel pair of interest (e.g., A-B, Region $1=$ R1) is filtered (convolved with a wavelet) for a specific frequency (e.g., $4 \mathrm{~Hz}$ ) to obtain a complex valued time series containing instantaneous amplitude (iv; $a_{n}$ ) and phase ( $\left(\phi_{n}\right)$ information (note: phase $\phi_{n}$ is shown here as cos $\phi_{n}$ ). $\boldsymbol{b}$, To quantify phase locking between two regions of interest (e.g., R1 and R2), the time-varying phase values $\left(\phi R 1_{n}\right.$ and $\left.\phi R 2_{n}\right)$ are first determined as in $\boldsymbol{a}$. For a given condition of interest the trial averaged $\mathrm{PLV}$ can be estimated at each time point following stimulus presentation. Across trials, the angular difference between the two phase series from R1 and R2 is calculated for the same time point with respect to stimulus onset. For a given time point $t_{T N+\tau}$ (where $N$ is trial number and $\tau$ is time from stimulus onset), the phase difference is calculated (e.g., $\phi R 1_{T 1+\tau}-\phi R 2_{T 1+\tau}$ for trial 1).c, Collecting the phase angle difference of each trial provides, for the time point of interest, a circular distribution of unit vectors in the complex plane, the average of which is the PLV (red lines indicate example trials 1-3 shown in $\boldsymbol{b}$; synthetic data). Therefore, the PLV is the circular mean (absolute vector length) of all the trials for that time point. $\boldsymbol{d}$, By repeating this calculation for each time point with respect to stimulus onset the time resolved PLV for a particular condition is constructed.

interelectrode correlations in amplitude or phase. To estimate amplitude and phase changes within and between RSC and MTL, the re-referenced data were transformed into time-frequency space using a wavelet approach (Le Van Quyen et al., 2001; Le Van Quyen and Bragin, 2007). A Morlet wavelet was convolved with the entire recorded time series from each bipolar montage to obtain a time-frequency decomposition, estimated using 5 cycles per frequency for the amplitude/phase at each time point ( $1 \mathrm{~Hz}$ steps; Theta: $3-8 \mathrm{~Hz}$; HFB: $70-180 \mathrm{~Hz}$ ). The resulting complex valued signal provides an instantaneous estimate of amplitude and phase for each center frequency. Changes in spectral power were calculated by taking the squared amplitude averaged across trials for the frequency range of interest, normalized to the mean of the entire power time series.

As commonly employed, phase-locking value (PLV) was used to estimate the synchronization between RSC and MTL (Lachaux et al., 1999) for each theta band center frequency (i.e., 3, 4, 5, 6, 7, and $8 \mathrm{~Hz}$ ). Figure 2 provides a schematic for the estimation of PLV. Briefly, PLV estimates the across-trial consistency of angular phase difference between two time series (regions 1 and 2, e.g., RSC and MTL; Fig. 2b), for a specified frequency and condition. Intertrial consistency of relative phase position between two regions is thought to reflect direct or mediated temporal coordination of functional relevance (Le Van Quyen and Bragin, 2007). For the PLV, the overall degree of phase consistency between two regions is defined as the circular mean (absolute vector length) of phase angle difference across all trials distributed in the complex plain (Fig. $2 c$ ). Therefore, in its raw form, a time-resolved PLV can be obtained for each condition, taking a range of $0-1$ (given the unit circle), where 1 is perfect phase locking (Fig. $2 c, d$ ). Given that the observed PLV at each time point is a singular value obtained by collapsing across trials, we used resampling statistics to obtain an estimate of PLV variance (error). Whereby, the PLV at each time point was recalculated using the same number of trials as the original calculation, however, trials were randomly selected with replacement (i.e., same trial can be selected multiple times) for each recalculation (Maris, 2012). This process was repeated 1000 times to provide a distribution of the PLV at each time point (used for error bars in Figs. 3 and 5).
As signal noise can produce non-zero PLV estimates, we subsequently used a standard randomization approach for phase-locking statistics (Lachaux et al., 1999) to compute a PLV significance threshold. To determine a chance level, we re-estimated the PLV between two regions by comparing experimental phase data (correctly ordered) in region 1 (e.g., RSC) to randomly selected data segments of equal trial duration from the second phase series (region 2; e.g., MTL). By shuffling the temporal relationship between phase series from the two regions of interest, one can iteratively build a distribution of chance PLV levels (i.e., characterize non-task noise factors contributing to intertrial phase consistencies). Therefore, we performed this shuffling procedure 200 times for each tested PLV pair, taking the maximum PLV of each iteration to build a surrogate distribution for comparison with the observed PLV. Identifying where the observed PLV falls within this surrogate (i.e., null) distribution allows for the direct assignment of a $p$ value (i.e., the probability of getting a value greater than the observed value given the null distribution) (Lachaux et al., 1999; Maris, 2012). Any electrode pairs with time points showing $p$ values $<0.05$ after stimulus presentation $(0-1500 \mathrm{~ms})$ were judged to be significant, and selected for further analysis.

\section{Results}

Subjects performed well on the task responding to all trials ( mean number of valid-trials: self-episodic $=47$, math $=48$, rest $=27)$. Mean reaction times were $2.3 \mathrm{~s}( \pm 0.90)$ for the self-episodic condition and $2.9 \mathrm{~s}( \pm 0.88)$ for the math condition. The rest condition had a fixed duration of $5 \mathrm{~s}$ with no response required. When considering true versus false judgments on average, the self-episodic condition contained more false than true judgments (true/false $=26 / 74 \%$ ), with comparable reaction times $(\mathrm{RT}$ true/false $=2.49 / 2.24 \mathrm{~s}$ ). Conversely, the math condition contained on average more true than false judgments (true/false $=75 / 25 \%$ ), with longer RTs for false trials (RT true/false $=2.64 / 3.62 \mathrm{~s}$ ). 
a
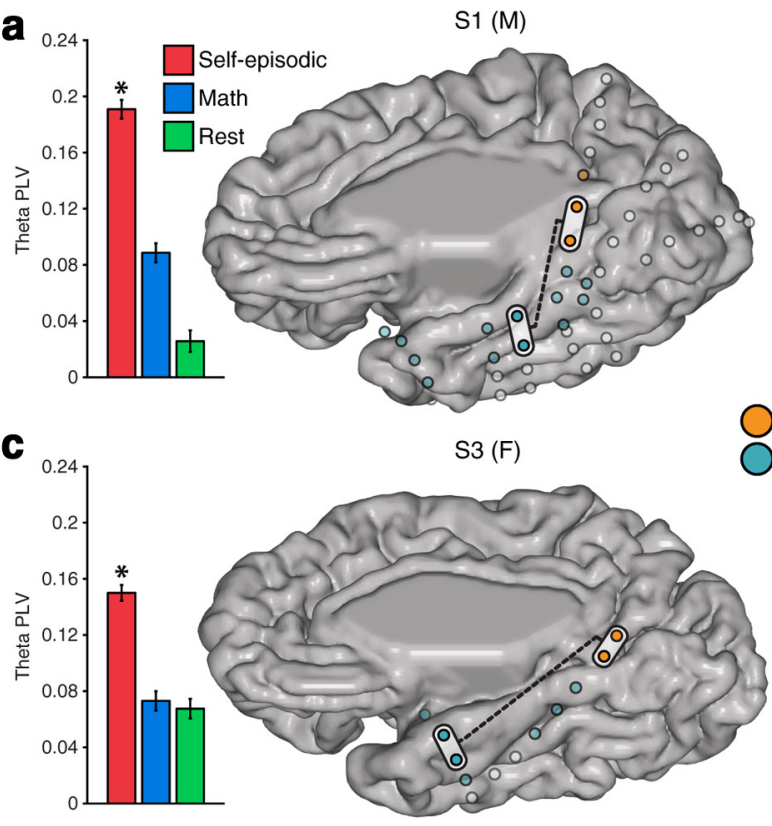

$\mathrm{S} 2(\mathrm{~F})$

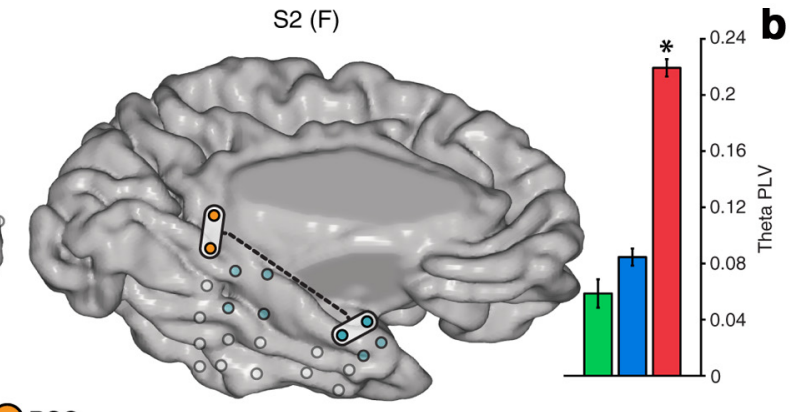

Oasc

d b

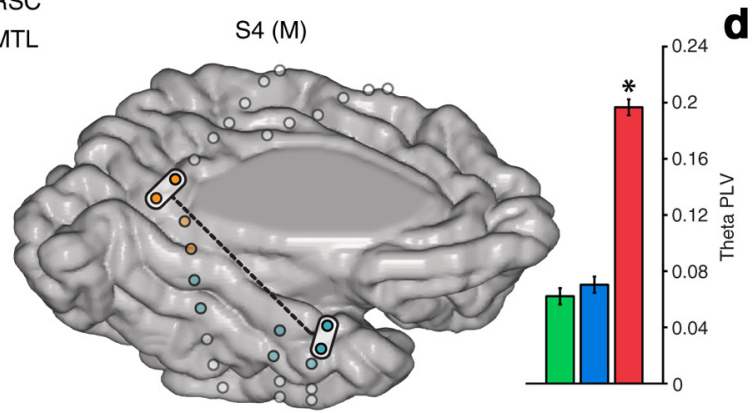


a

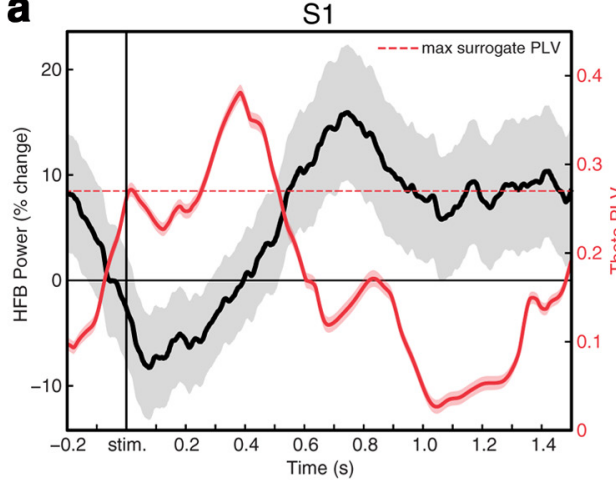

C

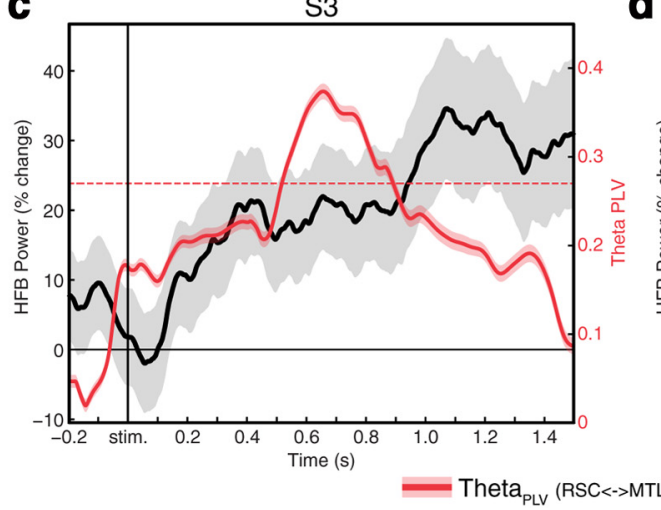

d b
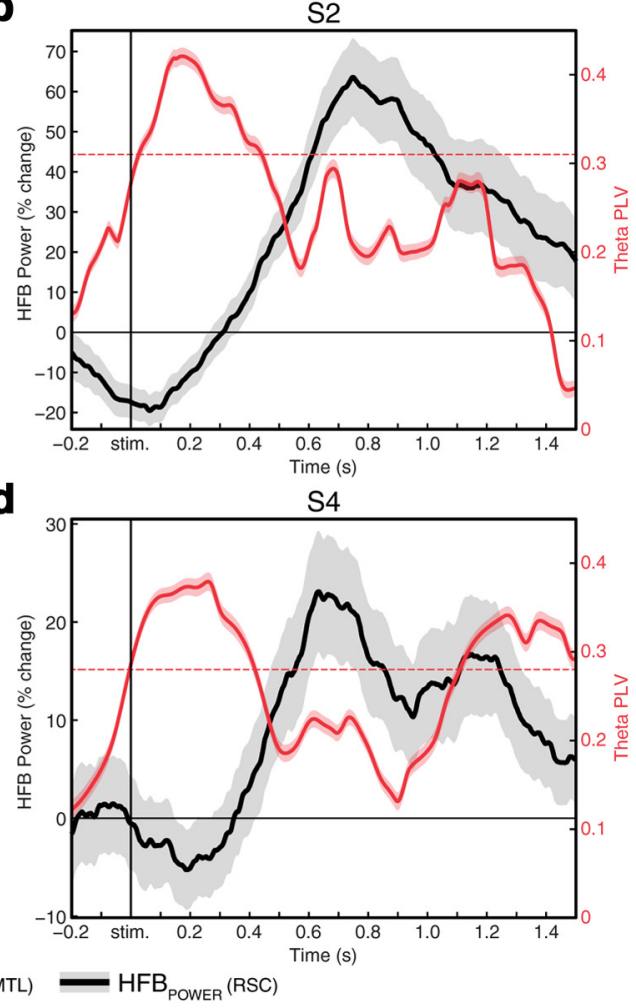

Figure 5. RSC-MTL theta phase locking precedes RSC HFB power. $\boldsymbol{a}$ - $\boldsymbol{d}$, Temporal dynamics of the raw theta band PLV for RSC-MTL coupling (red; right $y$-axis) and RSC HFB power (black; left $y$-axis) relative to stimulus (stim.) onset for the self-episodic condition across subjects ( $\mathrm{S} 1-\mathrm{S} 4$ ). Theta band PLV frequencies are $\mathrm{S1}=4 \mathrm{~Hz}, \mathrm{S2}=4 \mathrm{~Hz}, \mathrm{S3}=4 \mathrm{~Hz}$, and S4 $=3 \mathrm{~Hz}$ (see Results). Shading on both traces reflects SEM. Dashed red line $(\boldsymbol{a}-\boldsymbol{d})$ is the mean of maximal surrogate PLV values for each subject. Across subjects, theta band PLV between RSC-MTL transiently increases and peaks before the peak of RSC HFB activity.

therefore was not present during other conditions or other regions recorded. We focused our subsequent analyses on these significantly coupled RSC-MTL sites.

In identifying significant RSC-MTL pairs of theta phase locking during the self-episodic condition, we observed a strikingly consistent anatomical selectivity of interaction. In each subject, the MTL interaction only existed for the RSC pair that was shown to respond selectively and most strongly (in the HFB range) during autobiographical memory statements. For these RSC sites, HFB power was significantly greater for the self-episodic condition, when compared with either the math $(p=0.03$, Wilcoxon rank) or rest condition ( $p=0.05$, Wilcoxon rank) across subjects. Figure 4 shows the average change in HFB power for each condition across subjects (RSC and MTL). In S1 and S4, there were several electrodes abutting the RSC and nearby PMC, but only the electrode pair with the greatest HFB response during the autobiographical condition had significant phase locking with the MTL. Across subjects, theta phase coupling was seen between ventral RSC and anterior-mid MTL structures (Fig. 3). This finding is important because it suggests that the observed phase locking is not simply a function of proximal electrodes sharing measurement sources. During 3D electrode-brain reconstructions, we noted that the RSC electrode pairs in S1 and S2 were projected at the RSC/PHG border (i.e., one electrode seemed to be in the RSC while the other in the PHG). However, reviewing the $2 \mathrm{D}$ individual brain CTs suggested that these electrodes were within the RSC boundaries. It should also be noted that in S1 and S2, the adjacent PHG-only pairs did not produce any significant phase locking with anterior MTL pairs. Additionally, the RSC electrodes in S3 and S4 are clearly within the RSC borders (both on $2 \mathrm{D}$ images and $3 \mathrm{D}$ projections), and yet reproduced the same findings as S1 and S2, supporting the RSC origin of the phase locking effect.

Temporally, we also observed a consistent dynamic of theta phase locking across subjects. As shown in Figure 5, theta PLV is elevated early, but appears to have differing latencies of peak value between subjects. This latency and variation of peak theta phase locking is of great relevance to our previous observations regarding the latency of RSC HFB activation during episodic retrieval (Foster et al., 2012). Whereby, RSC HFB power typically had a late onset ( $\geq 400 \mathrm{~ms}$ ) and time to peak, but was early in response when compared with more dorsal regions of the PMC. Given this consistent delay in RSC activity, we previously hypothesized that relevant processes in the ventral and medial temporal lobe would precede RSC engagement (Foster et al., 2012). Despite this individual variability, when the RSC-MTL theta PLV time course is plotted together with the HFB power in RSC, a consistent temporal relationship is observed in each subject: The magnitude of phase locking between RSC-MTL consistently rises and peaks prior to the peak of RSC HFB power, with a typical lag of 300-400 ms between the two maxima. Therefore, before HFB activation, RSC displays a transient peak of phase locking in the theta band with anterior MTL cortices. It is important to note that a similar relationship was not observed for HFB power changes in coupled MTL pairs. HFB power change in MTL sites was not significantly different for the self-episodic condition compared with either the math ( $p=0.34$, Wilcoxon rank) or rest ( $p=0.69$, Wilcoxon rank) conditions across subjects (Fig. 4). In addition, we did not observe any significant differences in theta band power across conditions for either RSC or MTL (all $p>$ 
0.05, Wilcoxon rank). This temporal relationship also recapitulates the functional dependence noted above, which is that RSCMTL theta phase coupling was only observed for RSC sites displaying selective increases in HFB power to the self-episodic condition.

After testing our a priori hypothesis of selective theta band phase locking during the self-episodic condition, we then widened our frequency range of interest a posteriori for these identified pairs to see if any other frequencies displayed coupling (1-20 Hz, $1 \mathrm{~Hz}$ steps). Across subjects, theta phase locking for the self-episodic condition was maximal around $3-4 \mathrm{~Hz}$, when initially focusing on the $3-8 \mathrm{~Hz}$ range. As shown by the timefrequency example in Figure $6 a$, phase locking was indeed limited to this theta band range and typically not any other frequencies up to $20 \mathrm{~Hz}$. Across subjects, the distribution of mean PLV (surrogated controlled) was defined by the same $3-4 \mathrm{~Hz}$ peak within the theta band range, and also a more minor $8 \mathrm{~Hz}$ peak as shown in Figure $6 b$.

In light of this theta band specificity, we subsequently explored the temporal correlation between theta PLV and theta band power changes in MTL and RSC. For each subject we calculated a moving window correlation for RSC-MTL theta PLV and theta band power at both RSC and MTL for all conditions. Across all conditions and locations (RSC and MTL), there was no significant relationship between theta band power and theta band phase locking $(r=0.052, p=0.38)$. However, when considering each location separately, there were three significant correlations. Within RSC, theta band phase locking and theta band power showed a moderate positive correlation for the self-episodic condition $(r=0.43, p<0.008)$, and a negative correlation for the math condition $(r=-0.59, p<0.008)$ across subjects. Within the MTL, theta band phase locking showed a moderate negative correlation with theta band power during the math condition $(r=-0.42, p<0.008)$ across subjects. No other significant correlations for either region across conditions were observed (Bonferroni corrected).

\section{Discussion}

Using simultaneous recordings from human RSC and MTL cortex, we observed selective and transient theta band phase locking during autobiographical memory retrieval. Further analysis confirmed that this coupling was not seen for other studied conditions, other sites of recording, or other frequency ranges of interest. Anatomically, this coupling reflected ventral RSC and anterior MTL cortices. Temporally, theta phase coupling was maximal before peak activation of RSC, as indexed by increased HFB power, with a consistent temporal lag between the maxima of these two responses. Together, these findings provide the first electrophysiological evidence of a theta band-mediated interaction between the human RSC and MTL during conditions of RSC functional activation. These anatomical and temporal properties of the observed phase locking are consistent with the findings of a number of previous studies across species that have reported a strong relationship between the RSC and MTL (Vann et al., 2009). In addition, the theta band nature of this coupling also reflects a common oscillatory signature of interaction with the MTL. Therefore, our findings provide an important advance in studying human RSC function and its contribution to autobiographical memory retrieval via dynamic oscillatory interactions with the MTL.

Theta band oscillations have long been associated with memory processing in the MTL (Fell and Axmacher, 2011). We observed that theta phase locking was maximal around $3-4 \mathrm{~Hz}$
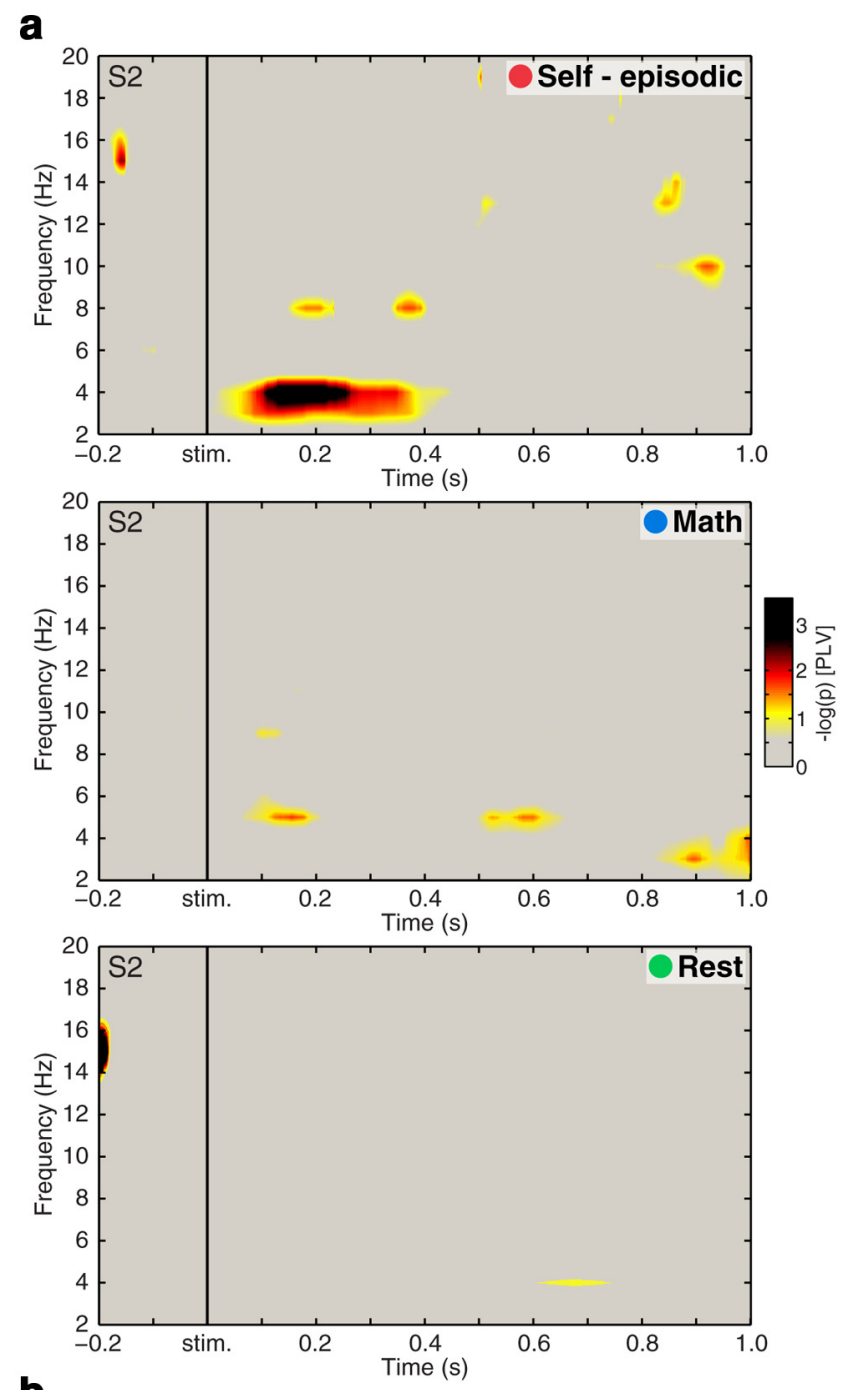

b

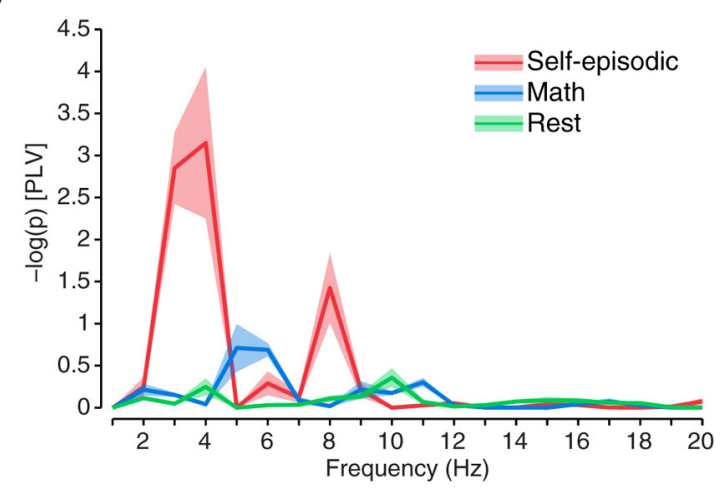

Figure 6. RSC-MTL phase locking is specific to the theta band range. $\boldsymbol{a}$, Example timefrequency plots (shown for $\mathrm{S2}$ ) of phase locking between RSC and MTL pairs for each condition (color map indicates $-\log (p)$ value based on comparison with surrogate data, e.g., $-\log (0.05)=2.99)$. Phase locking was specific to the theta band range and centered around 3- $4 \mathrm{~Hz}$, only for the self-episodic condition. $\boldsymbol{b}$, Distribution of mean (with SE) surrogate controlled PLV across all subjects (S1-S4) for each condition, estimated by averaging values from 0 to $500 \mathrm{~ms}$ after stimulus onset (note: $p$ values are lower than observed maximal values because of temporal averaging). Mean PLV distributions show a main 3-4 Hz peak and also a minor secondary $8 \mathrm{~Hz}$ peak for the self-episodic condition, whereas math and rest conditions are comparatively flat. 
specifically during autobiographical retrieval. This frequency range strongly overlaps with the intrinsic resting frequency of theta oscillations in the human PMC region, which has been shown to phase couple with HFB power at rest (Foster and Parvizi, 2012). Related to this observation, Lega et al. (2012) recently reported that across a large number of human hippocampal intracranial recordings, episodic memory effects were most associated with a $3 \mathrm{~Hz}$ theta oscillation. In addition, these authors also observed a faster $8 \mathrm{~Hz}$ theta rhythm in the hippocampus, which differs in its relationship to memory effects and spatial distribution, and is also detected in nearby temporal cortex (Lega et al., 2012). The observation of these two motifs bears some resemblance to our observations of a maximal peak at $3-4 \mathrm{~Hz}$ and a secondary minor peak at $8 \mathrm{~Hz}$ in the distribution of phase locking between RSC-MTL. We note that our data did not contain depth electrode recordings directly from the hippocampus proper, limiting a direct comparison. However, work in rodents has shown the preferred firing resonance within the ventromedial region of the entorhinal cortex to peak around $4 \mathrm{~Hz}$ (Giocomo et al., 2007). We also note, however, that the period of theta reverberations in the MTL may differ across species (Lega et al., 2012). With these important caveats in mind, our findings support the possibility of multiple theta band oscillatory mechanisms throughout the MTL and cortical memory systems that are of great interest for future investigations (Watrous et al., 2013).

Growing empirical evidence supports the role of field potential oscillations in modulating local spike timing (Jacobs et al., 2007; Fröhlich and McCormick, 2010; Anastassiou et al., 2011), and in coordinating interareal correlations of spiking activity (Fries, 2005; Womelsdorf et al., 2007; Canolty et al., 2010). The influence of theta oscillations upon the timing of spikes in the MTL, particularly hippocampal and rhinal cortices, is a striking and relevant example of spike-field interaction (Fell and Axmacher, 2011). While these interactions have chiefly been characterized in the rodent brain, evidence from intracranial recordings suggests a similar role in human memory function. For example, Rutishauser et al. (2010) reported significantly stronger theta range spike-field coherence in the human hippocampus for items subsequently remembered in a visual recognition paradigm.

In addition to local spike-field interactions, oscillatory activity can be synchronized between distal brain regions (Varela et al., 2001; Siegel et al., 2012). Such long-range correlations are often observed for slower frequency $(<40 \mathrm{~Hz})$ rhythms as phase-phase (matched frequency or integer multiple frequencies) or as phaseamplitude coupling (Canolty and Knight, 2010; Donner and Siegel, 2011; Fell and Axmacher, 2011). Temporal alignment of oscillatory activity may provide an efficient mechanism for coordinating spiking activity between distal regions by transiently synchronizing periodic oscillations to which local spiking displays some entrainment or temporal relationship (Fries, 2005; Canolty et al., 2010; Miller et al., 2012).

Given that the observed phase locking we report was transient and always proceeded the activation of RSC, one may conceive this to reflect a trigger event originating between the MTL and RSC leading to regional RSC functional activation in the HFB range (Foster et al., 2012). Such a transient influence may reflect an event of RSCMTL temporal alignment to improve inter-regional communication; however, it may also reflect the converse, allowing disengagement (misalignment) of the RSC for subsequent local processing (Donner and Siegel, 2011). It is therefore important to note that a variety of differing temporal activities may contribute to increased estimates of PLV, including phase resetting (i.e., intertrial phase locking/coherence for a given region; Tallon-Baudry et al., 1996; Delorme and Makeig, 2004).

Measures of phase synchrony or phase coherence, like the PLV, assume that temporal consistencies between two regions reflect a direct or mediated interaction of functional relevance. However, such techniques, while being computationally efficient, are subject to common limitations of multiple bivariate statistical testing and spatial sampling. Although surrogate data testing and other analytical developments can improve the identification of genuine interactions (Canolty et al., 2012), they still remain limited to the spatial sampling of recordings, particularly for human intracranial data. Therefore, the ability to directly assess the causal and directional nature of interareal connectivity requires more controlled methods. One opportunity for future study is the use of resting state corticocortical evoked potentials, and event-related electrical perturbation, to more sensitively probe the functional interaction between regions, like the RSC and MTL, with intracranial recordings (Matsumoto et al., 2007; Conner et al., 2011; Beauchamp et al., 2012).

As a subregion of the PMC, the RSC is part of a larger network of cortical regions that are activated during autobiographical retrieval, and are conversely suppressed during conditions of externally directed attention and working memory (Buckner et al., 2008). This wider default-mode network (DMN) has received a great deal of attention in cognitive neuroscience and neuroimaging, but its contributions to cognition are still unclear. Human intracranial electrophysiology can make unique contributions to understanding this network by providing precise measurements of neural population activity at the subsecond timescales over which cognition and behavior occurs. In addition to pronounced connectivity with the MTL, the RSC (as part of a larger PMC complex) shares strong interactions with other nodes of the DMN, such as the angular gyrus and medial prefrontal cortex (Fox et al., 2005; Power et al., 2011). Similar to the current study, it will be important for future investigations to electrophysiologically explore the interactions between the PMC and these nodes of the DMN to better understand how this system dynamically contributes to autobiographical memory retrieval and more broadly to human cognition.

\section{References}

Aggleton JP, Wright NF, Vann SD, Saunders RC (2012) Medial temporal lobe projections to the retrosplenial cortex of the macaque monkey. Hippocampus 22:1883-1900. CrossRef Medline

Anastassiou CA, Perin R, Markram H, Koch C (2011) Ephaptic coupling of cortical neurons. Nat Neurosci 14:217-223. CrossRef Medline

Beauchamp MS, Sun P, Baum SH, Tolias AS, Yoshor D (2012) Electrocorticography links human temporoparietal junction to visual perception. Nat Neurosci 15:957-959. CrossRef Medline

Bird CM, Burgess N (2008) The hippocampus and memory: insights from spatial processing. Nat Rev Neurosci 9:182-194. CrossRef Medline

Buckner RL, Andrews-Hanna JR, Schacter DL (2008) The brain's default network: anatomy, function, and relevance to disease. Ann N Y Acad Sci 1124:1-38. CrossRef Medline

Buzsáki G (2005) Theta rhythm of navigation: link between path integration and landmark navigation, episodic and semantic memory. Hippocampus 15:827-840. CrossRef Medline

Canolty RT, Knight RT (2010) The functional role of cross-frequency coupling. Trends Cogn Sci 14:506-515. CrossRef Medline

Canolty RT, Ganguly K, Kennerley SW, Cadieu CF, Koepsell K, Wallis JD, Carmena JM (2010) Oscillatory phase coupling coordinates anatomically dispersed functional cell assemblies. Proc Natl Acad Sci U S A 107: 17356-17361. CrossRef Medline

Canolty RT, Cadieu CF, Koepsell K, Ganguly K, Knight RT, Carmena JM (2012) Detecting event-related changes of multivariate phase coupling in dynamic brain networks. J Neurophysiol 107:2020-2031. CrossRef Medline 
Conner CR, Ellmore TM, DiSano MA, Pieters TA, Potter AW, Tandon N (2011) Anatomic and electro-physiologic connectivity of the language system: a combined DTI-CCEP study. Comput Biol Med 41:1100-1109. CrossRef Medline

Damasio AR (1989) Time-locked multiregional retroactivation: a systemslevel proposal for the neural substrates of recall and recognition. Cognition 33:25-62. CrossRef Medline

Dastjerdi M, Foster BL, Nasrullah S, Rauschecker AM, Dougherty RF, Townsend JD, Chang C, Greicius MD, Menon V, Kennedy DP, Parvizi J (2011) Differential electrophysiological response during rest, selfreferential, and non-self-referential tasks in human posteromedial cortex. Proc Natl Acad Sci U S A 108:3023-3028. CrossRef Medline

Delorme A, Makeig S (2004) EEGLAB: an open source toolbox for analysis of single-trial EEG dynamics including independent component analysis. J Neurosci Methods 134:9-21. CrossRef Medline

Donner TH, Siegel M (2011) A framework for local cortical oscillation patterns. Trends Cogn Sci 15:191-199. CrossRef Medline

Epstein RA (2008) Parahippocampal and retrosplenial contributions to human spatial navigation. Trends Cogn Sci 12:388-396. CrossRef Medline

Fell J, Axmacher N (2011) The role of phase synchronization in memory processes. Nat Rev Neurosci 12:105-118. CrossRef Medline

Foster BL, Parvizi J (2012) Resting oscillations and cross-frequency coupling in the human posteromedial cortex. Neuroimage 60:384-391. CrossRef Medline

Foster BL, Dastjerdi M, Parvizi J (2012) Neural populations in human posteromedial cortex display opposing responses during memory and numerical processing. Proc Natl Acad Sci U S A 109:15514-15519. CrossRef Medline

Fox MD, Snyder AZ, Vincent JL, Corbetta M, Van Essen DC, Raichle ME (2005) The human brain is intrinsically organized into dynamic, anticorrelated functional networks. Proc Natl Acad Sci U S A 102:9673-9678. CrossRef Medline

Fries P (2005) A mechanism for cognitive dynamics: neuronal communication through neuronal coherence. Trends Cogn Sci 9:474-480. CrossRef Medline

Fröhlich F, McCormick DA (2010) Endogenous electric fields may guide neocortical network activity. Neuron 67:129-143. CrossRef Medline

Giocomo LM, Zilli EA, Fransén E, Hasselmo ME (2007) Temporal frequency of subthreshold oscillations scales with entorhinal grid cell field spacing. Science 315:1719-1722. CrossRef Medline

Greicius MD, Supekar K, Menon V, Dougherty RF (2009) Resting-state functional connectivity reflects structural connectivity in the default mode network. Cereb Cortex 19:72-78. Medline

Hermes D, Miller KJ, Noordmans HJ, Vansteensel MJ, Ramsey NF (2010) Automated electrocorticographic electrode localization on individually rendered brain surfaces. J Neurosci Methods 185:293-298. CrossRef Medline

Jacobs J, Kahana MJ, Ekstrom AD, Fried I (2007) Brain oscillations control timing of single-neuron activity in humans. J Neurosci 27:3839-3844. CrossRef Medline

Jerbi K, Vidal JR, Ossandon T, Dalal SS, Jung J, Hoffmann D, Minotti L, Bertrand O, Kahane P, Lachaux JP (2010) Exploring the electrophysiological correlates of the default-mode network with intracerebral EEG. Front Syst Neurosci 4:27. Medline

Kobayashi Y, Amaral DG (2003) Macaque monkey retrosplenial cortex: II. Cortical afferents. J Comp Neurol 466:48-79. CrossRef Medline

Lachaux JP, Rodriguez E, Martinerie J, Varela FJ (1999) Measuring phase synchrony in brain signals. Hum Brain Mapp 8:194-208. CrossRef Medline

Lachaux JP, Rudrauf D, Kahane P (2003) Intracranial EEG and human brain mapping. J Physiol Paris 97:613-628. CrossRef Medline

Lachaux JP, Axmacher N, Mormann F, Halgren E, Crone NE (2012) Highfrequency neural activity and human cognition: past, present and possible future of intracranial EEG research. Prog Neurobiol 98:279-301. CrossRef Medline

Le Van Quyen M, Bragin A (2007) Analysis of dynamic brain oscillations: methodological advances. Trends Neurosci 30:365-373. CrossRef Medline
Le Van Quyen M, Foucher J, Lachaux J, Rodriguez E, Lutz A, Martinerie J, Varela FJ (2001) Comparison of Hilbert transform and wavelet methods for the analysis of neuronal synchrony. J Neurosci Methods 111:83-98. CrossRef Medline

Lega BC, Jacobs J, Kahana M (2012) Human hippocampal theta oscillations and the formation of episodic memories. Hippocampus 22:748-761. CrossRef Medline

Margulies DS, Vincent JL, Kelly C, Lohmann G, Uddin LQ, Biswal BB, Villringer A, Castellanos FX, Milham MP, Petrides M (2009) Precuneus shares intrinsic functional architecture in humans and monkeys. Proc Natl Acad Sci U S A 106:20069-20074. Medline

Maris E (2012) Statistical testing in electrophysiological studies. Psychophysiology 49:549-565. CrossRef Medline

Matsumoto R, Nair DR, LaPresto E, Bingaman W, Shibasaki H, Lüders HO (2007) Functional connectivity in human cortical motor system: a cortico-cortical evoked potential study. Brain 130:181-197. Medline

Miller KJ, Weaver KE, Ojemann JG (2009) Direct electrophysiological measurement of human default network areas. Proc Natl Acad Sci U S A 106:12174-12177. CrossRef Medline

Miller KJ, Foster BL, Honey CJ (2012) Does rhythmic entrainment represent a generalized mechanism for organizing computation in the brain? Front Comput Neurosci 6:85. Medline

Niedermeyer E, Lopes da Silva FH (2005) Electroencephalography: basic principles, clinical applications, and related fields, Ed 5. Philadelphia: Lippincott Williams and Wilkins.

Nunez PL, Srinivasan R, Westdorp AF, Wijesinghe RS, Tucker DM, Silberstein RB, Cadusch PJ (1997) EEG coherency. I: Statistics, reference electrode, volume conduction, Laplacians, cortical imaging, and interpretation at multiple scales. Electroencephalogr Clin Neurophysiol 103:499-515. CrossRef Medline

Ossandón T, Jerbi K, Vidal JR, Bayle DJ, Henaff MA, Jung J, Minotti L, Bertrand O, Kahane P, Lachaux JP (2011) Transient suppression of broadband gamma power in the default-mode network is correlated with task complexity and subject performance. J Neurosci 31:14521-14530. CrossRef Medline

Parvizi J, Van Hoesen GW, Buckwalter J, Damasio A (2006) Neural connections of the posteromedial cortex in the macaque. Proc Natl Acad Sci U S A 103:1563-1568. CrossRef Medline

Power JD, Cohen AL, Nelson SM, Wig GS, Barnes KA, Church JA, Vogel AC, Laumann TO, Miezin FM, Schlaggar BL, Petersen SE (2011) Functional network organization of the human brain. Neuron 72:665-678. CrossRef Medline

Ranganath C, Ritchey M (2012) Two cortical systems for memory-guided behaviour. Nat Rev Neurosci 13:713-726. CrossRef Medline

Rutishauser U, Ross IB, Mamelak AN, Schuman EM (2010) Human memory strength is predicted by theta-frequency phase-locking of single neurons. Nature 464:903-907. CrossRef Medline

Siegel M, Donner TH, Engel AK (2012) Spectral fingerprints of large-scale neuronal interactions. Nat Rev Neurosci 13:121-134. Medline

Tallon-Baudry C, Bertrand O, Delpuech C, Pernier J (1996) Stimulus specificity of phase-locked and non-phase-locked $40 \mathrm{~Hz}$ visual responses in human. J Neurosci 16:4240-4249. Medline

Vann SD, Aggleton JP, Maguire EA (2009) What does the retrosplenial cortex do? Nat Rev Neurosci 10:792-802. CrossRef Medline

Varela F, Lachaux JP, Rodriguez E, Martinerie J (2001) The brainweb: phase synchronization and large-scale integration. Nat Rev Neurosci 2:229-239. CrossRef Medline

Watrous AJ, Tandon N, Conner CR, Pieters T, Ekstrom AD (2013) Frequency-specific network connectivity increases underlie accurate spatiotemporal memory retrieval. Nat Neurosci 16:349-356. CrossRef Medline

Weiner KS, Grill-Spector K (2013) Neural representations of faces and limbs neighbor in human high-level visual cortex: evidence for a new organization principle. Psychol Res 77:74-97. CrossRef Medline

Womelsdorf T, Schoffelen JM, Oostenveld R, Singer W, Desimone R, Engel AK, Fries P (2007) Modulation of neuronal interactions through neuronal synchronization. Science 316:1609-1612. CrossRef Medline 